\title{
Deutschlands Krisenmanagement in der CORONA-Pandemie. Herausforderungen eines föderalen politisch-administrativen Systems
}

\section{CORONA-Krisenmanagement in Deutschland. Herausforderungen und Resultate}

Im Zuge der weltweiten CORONA-Pandemie sind auch in Deutschland das politisch-administrative System, das Gesundheitssystem, die Wirtschaft, der Sozialstaat und auch die Gesellschaft seit Anfang des Jahres 2020 massiv unter Druck geraten. Als weltweit vernetztes Land mit stark exportabhängiger Wirtschaft, aber auch als wichtiger Mitgliedstaat der EU steht Deutschland beim CORONA-Krisenmanagement damit vor der größten Herausforderung seit dem Ende des Zweiten Weltkriegs vor 75 Jahren und einer politisch-administrativen und gesellschaftlichen Bewährungsprobe ohne Vorbild (siehe auch Franzke, 2020).

Bei der Bewältigung der COVID-19-Pandemie gewinnt das politisch-administrative Krisenmanagement an Bedeutung, d. h. alle Maßnahmen zur Prävention, Erkennung, Bewältigung und Nachbereitung bereits eingetretener Katastrophen. Zu einem effektiven und effizienten Krisenmanagement gehört die Schaffung konzeptioneller, organisatorischer und verfahrensmäßiger Voraussetzungen, ,die eine schnellstmögliche Zurückführung einer eingetretenen außergewöhnlichen Situation in den Normalzustand unterstützen“" (Runderlass des NRW-Ministeriums für Inneres und Kommunales 2016, Art 1.). Es wird sich zeigen, ob nach dieser Pandemie eine Rückkehr zum ex ante Zustand überhaupt möglich sein wird.

\section{Föderalismus}

Das politisch-administrative System Deutschlands ist geprägt durch einen ,,unitarische Föderalismus“ (Hesse, 1962), der - nach Manfred G. Schmidt ,so viel Gleichwertigkeit bei der politischen Regulierung und den Lebensverhältnissen sein eigen nennt, dass man meinen könnte, er sei ein dezentraler Einheitsstaat" (Schmidt, 2015, S. 1). Dieser ist geprägt durch die Existenz von zwei politisch eigenständigen Ebenen (Bund und Länder) und drei eigenständigen Verwaltungsebenen (Bund, Länder und Kommunen). Die Kommunalverwaltungen sind Teil der jeweiligen Landesverwaltung und keine eigenständige politische Ebene.

Der unitarische Föderalismus basiert auf zwei potenziell gegensätzliche Prinzipien: Einerseits genießen die 16 Bundesländer eine starke Position und Autonomie in Bezug 
auf ihre eigene Regierung, Gesetzgebung, Verwaltung und Justizbehörden sowie ein hohes $\mathrm{Ma} ß$ an Diskretion, insbesondere in Bezug auf die Umsetzung von (Bundes-, Landes- und EU-) Gesetzen. Andererseits ist die Einheitlichkeit von Recht, Wirtschaft und Lebensbedingungen in Deutschland verfassungsrechtlich geschützt.

Mehrere etablierte Mechanismen ermöglichen die Zusammenarbeit und gemeinsame Entscheidungsfindung über alle Ebenen des multilevel governance systems, um die Einheit des Bundes zu gewährleisten (siehe Fuhr et al., 2018; Kuhlmann, 2020; Behnke, 2020). Es wurden vielfältige zwischenstaatliche Kooperationsmechanismen institutionalisiert, von denen einige nur die Länder betreffen (horizontale Zusammenarbeit), während andere Bund und Länder betreffen (vertikale Zusammenarbeit). In diesem Umfeld ist das Prinzip eines „Exekutivföderalismus“ ein weiteres wichtiges Schlüsselmerkmal deutscher Politikkoordinierung. Nach diesem Prinzip ist die Bundesebene hauptsächlich für die Formulierung der Politik verantwortlich, während die Länder und Kommunen hauptsächlich an der Umsetzung der Politik beteiligt sind (Kuhlmann, Wollmann, 2019).

\section{Corona-Krisenmanagement in Deutschland}

In Deutschland wird die Pandemiebekämpfung also von einem föderalen politischadministrativen System geleistet. Die theoretische politikwissenschaftliche Fragestellung dieses Beitrages bezieht sich daher darauf, wie sich diese dezentrale, föderale Struktur auf die Pandemiebekämpfung ausgewirkt hat. Sind hier Vorteile gegenüber unitarischen Systemen erkennbar oder eher Nachteile?

Deutschland hat bislang in der Pandemie seine vorhandenen verfassungsrechtlichen Notstandsregelungen nicht genutzt, bei einer Zuspitzung der CORONA-Lage, bleibt es weiterhin möglich, in den Katastrophenmodus zu wechseln und z. B. die Katastrophenschutzgesetze der Länder anzuwenden. Rechtliche Basis für die CoronaMaßnahmen bildet in Deutschland die sehr weit gefasste allgemeine Generalklausel des nationalen Infektionsschutzgesetzes, wonach Behörden alle „notwendigen Schutzmaßnahmen“ verhängen können, ,soweit und solange es zur Verhinderung der Verbreitung übertragbarer Krankheiten erforderlich ist" (IfSG 2020, § 28 Abs. 1). Ergänzt wurde diese Rechtsgrundlage durch das 27. März 2020 in Kraft getretenen „Gesetz zum Schutz der Bevölkerung bei einer epidemischen Lage von nationaler Tragweite”, die der Bundestag zwei Tage vorher festgestellt aktuell hatte. Damit kann das Bundesgesundheitsministerium bundesweit und ohne Zustimmung des Bundesrates Anordnungen im (normalerweise föderalen) Gesundheitswesen treffen, was es in einer Reihe von Fällen seither aus getan hat.

Im Rahmen des kooperativen Föderalismus sind der Bund und die 16 Länder auch bei der Pandemie-Bekämpfung vielfältig miteinander verflochten und verschränkt. Die Bundesregierung trägt die nationale Verantwortung für die Pandemie-Bekämpfung, benötigt dazu aber administrative Unterstützung der Länder. Diese sind wiederum für die Gefahrenabwehr im Katastrophenfall gemäß Artikel 70 des Grundgesetzes verantwortlich. Daher werden alle wichtigen Entscheidungen in der CORONA-Krise durch die Bundeskanzlerin und die Regierungschefs der Länder gemeinsam diskutiert und 
beschlossen. Bei deren Umsetzung kann es dabei durchaus regionale Unterschiede geben, die von den jeweiligen Ländern politisch zu verantworten sind. Diese ergeben sich aus differenzierten COVID-19 Infektionslagen bzw. aus unterschiedlichen Politikansätzen bei der Bekämpfung der Pandemie.

Bund und Länder verfolgt mit ihrem Handeln in der CORONA-Krise drei grundsätzliche Ziele: (1) Die Gesundheit der Bevölkerung zu schützen und die Leistungsfähigkeit des Gesundheitssystems zu bewahren (Infektionsgeschehen verlangsamen, Gesundheitswesen stärken); (2) die Folgen der Pandemie für Bürger, Beschäftigte und Unternehmen abfedern (Bürger umfassend unterstützen, Wirtschaft stabilisieren und Arbeitsplätze schützen) sowie (3) die Pandemie in internationaler Zusammenarbeit bewältigen (Europäische Solidarität und internationale Zusammenarbeit stärken) (Presse- und Informationsamt der Bundesregierung 2020a). Als primäre Herausforderungen in der COVID-19-Pandemie galt vor allem am Beginn der Pandemie, deren Ausbreitung zu verlangsamen, damit das deutsche Gesundheitssystem nicht überfordert oder handlungsunfähig wird. Bis heute gelang es, dieses Ziel zu erreichen.

Im Folgenden soll kurz auf die Schwerpunkte der Pandemiebekämpfung auf der Ebene des Bundes, der Länder und der Kommunen eingegangen werden.

\section{Bundesebene}

Am Anfang der Pandemie und seit Anfang Mai 2020 versucht die Bundesregierung ihr Krisenmanagement innerhalb des exekutiven Normalbetriebes zu gestalten. Während des Shutdowns von Mitte März bis Anfang Mai 2020 handelte die Bundesregierung in einem besonderen Krisenmodus (Siehe Bundesregierung 2020a). Immer montags fand das sogenannte „Kleine Corona-Kabinett“ unter Leitung der Bundeskanzlerin statt, an dem die Minister der Verteidigung, der Finanzen, des Inneren, des Auswärtiges, für Gesundheit und der Chef des Bundeskanzleramtes teilnahmen. Nach der regulären Kabinettssitzung jeweils am Mittwoch traf sich immer Donnerstag das sogenannte „Große Corona-Kabinett“, zu dem neben den Teilnehmern des „Kleinen Corona-Kabinetts“ zusätzlich jene Fachminister hinzugeladen wurden, die bei den zu behandelnden Themen zuständig sind.

Auf der Staatssekretärsebene bestand eine ähnliche Struktur. Montags trafen sich die beamteten Staatssekretäre der Bundesministerien unter Leitung des Chefs des Bundeskanzleramtes Helge Braun. Gemeinsam bereiteten sie die reguläre Sitzung des Regierungskabinetts am Mittwoch vor, wobei die einzelnen Ressorts Themen anmelden konnten, die aufgrund der Epidemie sofort bewältigt werden müssen. Die Staatssekretäre legen fest, mit welcher personellen Zusammensetzung und bis wann die Anliegen bearbeitet sein sollen. Mittwochs fand eine zusätzliche Runde der beamteten Staatssekretäre (alle Ressorts) unter Leitung des Chefs des Bundeskanzleramtes statt. Diese bereitete die Beschlüsse des großen Corona-Kabinetts vor und legte zu weiteren Themen den Bearbeitungsgang fest.

Das Bundesgesundheitsministerium sowie das Ressort des Inneren und Sport hatten einen gemeinsamen Krisenstab etabliert, wo weitere operative Themen direkt bearbeitet werden konnten. Dazu gehören unter anderem die praktische Umsetzung der 
Beschlüsse der Corona-Kabinette, die Befassung mit Hilfeersuchen der Länder sowie die Erstellung von Lagebildern zur Information der Bundesregierung und der Öffentlichkeit. Im Zuständigkeitsbereich des Bundesgesundheitsministeriums lag auch der sogenannte Beschaffungsstab. Dazu zählten auch Experten des Finanzministeriums und des Auswärtigen Amtes. Neben den Ministeriumsvertretern gehören zum Beschaffungsstab außerdem Verbindungspersonen deutscher Unternehmen mit internationalem Geschäft, mit denen das Bundesgesundheitsministerium Rahmenvereinbarungen geschlossen hat.

Anfang Mai wurde dieser Krisenmechanismus der Bundesregierung, insbesondere was das Große und Kleine Corona-Kabinett betrifft, beendet. Wegen der ansteigenden Infektionszahlen wird in der Bundesregierung Mitte August 2020 über eine Reaktivierung der Corona-Kabinette nachgedacht.

\section{Länderebene}

Auch auf Länderebene wurden Corona-Krisenstäbe etabliert. Im Bundesland Brandenburg wurde z. B. am 14.3. ein „Interministerielle Koordinierungsstab Coronavirus der Landesregierung“ unter Leitung des Gesundheitsstaatssekretärs gebildet, der anfangs rund um die Uhr im Koordinierungszentrum Krisenmanagement (KKM) des Innen- und Kommunalministeriums tagte (Siehe Brandenburgisches Ministerium für Soziales, Gesundheit, Integration und Verbraucherschutz 2020). Koordiniert wird die Zusammenarbeit zwischen dem Ministerium für Soziales, Gesundheit, Integration und Verbraucherschutz und dem Ministerium des Innern und für Kommunales, die beide wichtige Aufgaben bei der Pandemiebekämpfung haben. Informationen vom Bund und anderen Ländern wurden hier gebündelt und gesteuert. Die aktuelle Lage wird mit allen Verantwortlichen bewertet, und notwendige Maßnahmen im Land abgestimmt. Ein tägliches Lagebild „Corona“ wird veröffentlicht sowie Telefonschaltkonferenzen mit den Landkreisen und kreisfreien Städten veranstaltet. Seit Mitte Juni werden die Länderkrisenstäbe heruntergefahren bzw. geschlossen (z. B. in Thüringen und Brandenburg) und deren Aufgaben wieder in den administrativen Normalbetrieb überführt.

\section{Kommunale Ebene}

Die kommunale Autonomie ist in Deutschland verfassungsrechtlich geschützt; zugleich sind die Kommunen Bestandteil der jeweiligen Landesverwaltung. Die überörtliche kommunale Ebene in Deutschland bilden aktuell 294 Landkreise $^{1}$ und 107 kreisfreien Städte ${ }^{2}$. Die örtliche kommunale Ebene besteht aktuell aus 10.795 Städten und Gemeinden, wovon ca. ein Drittel verbandsfrei sind d. h. über eine eigene Verwaltung

${ }^{1}$ Die Landkreise sind sowohl Institution der kommunalen Selbstverwaltung als auch mit dem Landrat an ihrer Spitze in den meisten Ländern die untere staatliche Verwaltungsbehörde.

${ }^{2}$ Die kreisfreien Städte erledigen städtische und kreisliche Aufgaben gemeinsam in eigener Verantwortung. Zu den kreisfreien Städten gehören auch Berlin und Hamburg, die zugleich Bundesländer sind. 
verfügen. ${ }^{3}$ Die kommunalen Spitzenverbände (Deutscher Städtetag, Deutscher Städteund Gemeindebund sowie Deutscher Landkreistag) spielen in der CORONA-Krise als Stimme der Kommunen auf Bundes- und Länderebene eine erhebliche Rolle.

Der Kern der Pandemie-Krisenbewältigung liegt auf Ebene der Kreise und kreisfreien Städte. Um die jeweiligen Hauptverwaltungsbeamten (Landräte auf Kreisebene und Oberbürgermeister in den kreisfreien Städten) bei der lokalen Pandemiebekämpfung zu unterstützen sowie die interne Koordinierung und Steuerung der jeweiligen Kommunalverwaltung zu gewährleisten, wurden seit Mitte Februar in allen Landkreisen und kreisfreien Städten Deutschlands Corona-Krisenstäbe gebildet. ${ }^{4}$ Eigentlich waren es sogar zwei Stäbe, denn für den Fall, dass der Krisenstabes durch eine Corona-Infektion in seinen Reihen in Quarantäne gehen muss, wurde oftmals (z. B. in der Stadt Wittenberge) ein zweiter Stab im Home-Office vorgehalten.

Der Krisenstab ist dem jeweiligen Hauptverwaltungsbeamten unterstellt und hat vornehmlich Bündelungs- und Koordinierungsfunktion. Dort werden die kreislichen Entscheidungen zur Eindämmung der COVID-19-Pandemie getroffen, Ablaufpläne erstellt und Szenarien entwickelt. Die Krisenstäbe organisieren z. B. die zeitweilige Umsetzung von Mitarbeitern, überwiegend ins Gesundheitsamt. Sie führen alle verfügbaren Informationen zum Kreisgebiet zusammen, werten diese aus und verteilen diese innerhalb der Kreisverwaltung und an andere wichtige Adressaten im Landkreis. Auch die regionale Öffentlichkeit wird über die Pressestelle und die Bürgerservices informiert. Zu den wichtigsten Aufgaben hat sich die Beschaffung persönlicher Schutzausstattung wie Atemschutzmasken, Einweganzügen und Desinfektionsmitten entwickelt. Die Landkreise sollen diese eigentlich von den Ländern zugeteilt bekommen, deren Lieferungen reichten aber zumeist nicht aus. Daher müssen sie sich selber kümmern und diese Ausrüstung selbst besorgen sowie dann an die kreislichen Gesundheitseinrichtungen weiterzuleiten.

Seit dem 20.4. wurde in einzelnen Kommunen begonnen, die Ressourcen der Stäbe herunterzufahren. Eine Reaktivierung bleibt aber jederzeit möglich, was auch in einzelnen Corona-Hotspots bereits erfolgt ist. Anfang August 2020 haben wegen lokal ansteigender Infektionszahlen einige kommunale Krisenstäbe ihre Arbeit wieder aufgenommen.

\section{Bisheriger Verlauf der CORONA-Krise in Deutschland}

Die CORONA-Krise traf Deutschland wie alle anderen Länder der Welt unvermittelt. Die innenpolitische Situation in Deutschland veränderte sich seit Ausbruch der

${ }^{3}$ Die restlichen Gemeinden gehören einem Amt oder einer Verwaltungsgemeinschaft an, bei der die lokalen Verwaltungen zentralisiert und bestimmte lokale Aufgaben konzentriert sind.

${ }^{4}$ Die personelle Zusammensetzung der kommunalen CORONA-Krisenstäbe variiert. Hier als Beispiel die Zusammensetzung des Krisenstabes im Rhein-Kreises (NRW): Amt für Sicherheit und Ordnung, Gesundheitsamt, Schulamt, Jugendamt, Sozialamt, Personalamt, Controlling-Abteilung, Kommunalaufsicht, Pressestelle und alle Dezernenten der Kreisverwaltung. Ständige Mitglieder sind zudem der Ärztliche Leiter Rettungsdienst, der Kreisbrandmeister, die Kreisleitstelle und das Kreisverbindungskommando der Bundeswehr. Je nach Bedarf werden weitere externe Experten eingebunden, z. B. aus den Gemeinden des Landkreises, den Krankenhäusern oder der Polizei (Homepage des Landkreises, abgerufen am 23.4.2020). 
Pandemie im Dezember 2019 so rasch, dass es sinnvoll erscheint, die einzelnen Etappen und die dabei erkennbaren politischen Konzepte des Bundes und der Länder beim Umgang mit der Pandemie an dieser Stelle erstmal kurz darzustellen.

\section{Phase I - Covid-19 erreicht Deutschland}

Deutschland hat auf die Corona-Pandemie, die wahrscheinlich im Dezember 2019 in China ausbrach, erst spät reagiert. Am 27.1.2020 wurde in Deutschland die ersten beiden Patienten mit COVID-19 im Bundesland Bayern registriert, die durch Infektion mit dem Coronavirus SARS-CoV-2 ausgelöst wird. Das Risiko für die Bevölkerung wurde zu diesem Zeitpunkt und noch bis Ende Februar sowohl von den politisch Verantwortlichen im Bund und Ländern als auch vom Robert-Koch-Institut (RKI) als zentraler Bundesbehörde zur Krankheitsüberwachung und -prävention als ,gering bis mäßig“ eingeschätzt. Am 2.3. wurde dies auf „mäßig“ erhöht. Ende Februar bildet sich der erste deutsche COVID-19-Hotspot im Kreis Heinsberg (Bundesland NordrheinWestfalen). Nun erreichte die Corona-Krise erstmals bundesweite öffentliche Aufmerksamkeit. Am 26.2. schloss der Landkreis Heinsberg Schulen und Kindergärten für eine Woche. Dies war die erste administrative Regulierung in Deutschland in der CORONA-Pandemie, die die Bevölkerung unmittelbar betraf.

Nach diesem Ausbruch des Virus im Kreis Heinsberg begann sich der politischadministrative Umgang mit COVID-19 in Deutschland rasch zu verändern. Am 27.2. richtet die Bundesregierung den oben schon genannten gemeinsamen CORONAKrisenstab unter Federführung des Innen- und Gesundheitsministeriums ein, aus dem später das CORANA-Kabinett hervorgehen wird. Bis Mitte März richten auch alle Landesregierungen solche Krisenstäbe ein, um den Schutz der Bevölkerung sicherstellen. In diesen Tagen versucht die Bundesregierung in ihrem Umgang mit der Pandemie „Maß und Mitte“ zu finden. Der öffentliche Druck nahm zu, als ab 8. März in Deutschland vermehrt positiv getestete Covid-19-Fälle bei der Rückreise aus dem Ski-Urlaub in Tirol (Österreich) gemeldet werden. Am 9. März wird von den ersten beiden Todesfällen im Zusammenhang mit Covid-19 in Deutschland berichtet.

\section{Phase II - Shutdown}

Am 11. März macht Bundeskanzlerin Merkel auf einer Pressekonferenz die Eindämmung des COVID-19 Virus in Deutschland zur „Chefsache“ und betont: „Das Virus ist in Europa angelangt ... Die Maßstäbe für unser politisches Handeln, wie wir auf ein Virus reagieren, das wir noch nicht ausreichend kennen, für das wir keine Therapie und ... keinen Impfstoff haben, ergeben sich aus dem, was uns Wissenschaftler und Experten sagen" (Pressekonferenz von Bundeskanzlerin Merkel, Bundesgesundheitsminister Spahn und RKI-Chef Wieler 2020). Die Wissenschaft, speziell einige Virologen und Epidemiologen, erlangen rasch zentralen Einfluss auf die Entscheidungen der Bundesregierung in der CORONA-Krise. Als kurzfristiges Handlungsziel beschreibt die Bundeskanzlerin, die Ausbreitung des Virus zu verlangsamten, um Zeit zu 
gewinnen, damit das deutsche Gesundheitssystem nicht überlastet wird. Notwendig sei dabei ein abgestimmtes Handeln aller staatlicher Ebenen, um bei gleicher Situation möglichst in allen Bundesländern gleich zu handeln, was aber nur annähernd gelingt. Merkel unterstreicht: „... wir tun wirklich gut daran, von der Bundesebene bis zur lokalen Ebene den Empfehlungen des Robert Koch-Instituts nachzukommen“ (Pressekonferenz von Bundeskanzlerin Merkel, Bundesgesundheitsminister Spahn und RKIChef Wieler 2020). Am 17.3. stufte das RKI das Risiko der COVID-19-Pandemie für die Bevölkerung in Deutschland erstmals als ,hoch“ ein.

Am 12.3. berät sich Bundeskanzlerin Merkel zum für längere Zeit letzten Mal direkt mit den Ministerpräsidenten der Länder. Die Tagung steht unter dem Eindruck der apokalyptischen Bilder nächtlicher Leichentransporte von COVID-19-Opfern im dem italienischen Bergamo und der Ankündigung eines Shutdowns in Frankreich. Bund und Länder beschließen, ab dem 16.3. soziale Kontakte weiter zu beschränken und alle Kindergärten, Schulen, Hochschulen sowie Universitäten bis auf weiteres zu schließen (Presse- und Informationsamt der Bundesregierung (2020b). Am nächsten Tag beginnen die Bundesländer mit der Schließung weiterer öffentlicher und privater Einrichtungen. Demonstrationen werden verboten ebenso wie religiöse Zeremonien. Am 22. März verschärfen Bund und Länder die Beschränkung sozialer Kontakte weiter (Siehe Bundesregierung 2020b). Ab dem 23. März wird Deutschland zum ersten Mal in seiner Geschichte in eine Quarantäne versetzt (Shutdown), die durch weitreichende Ausgangsbeschränkungen für die Bevölkerung gewährleistet werden soll. Viele öffentliche Einrichtungen werden geschlossen. Der Notstand wurde in Deutschland allerdings nicht ausgerufen. Deutschland wechselte in den politisch-administrativen Corona-Krisen-Modus mit absehbaren dramatischen Folgen in Wirtschaft und Gesellschaft. Es war die Stunde der Exekutiven in Bund und Ländern.

Faktisch wurden damit viele Grundrechte der Verfassung suspendiert. Anfangs wurde dies von der Bevölkerung angesichts der Pandemielage hingenommen, seit Anfang April nimmt der öffentliche Druck aber stark zu, die Einschränkungen von Grundrechten immer wieder zu überprüfen und möglichst rasch wieder abzubauen. Anfangs hielten die meisten der angerufenen Verwaltungsgerichte diese Generalklausel angesichts der außergewöhnlichen Lage für ausreichend, um die in der CORONA-Krise getroffenen Maßnahmen zu stützen. Am 28.4. hat der Saarländische Verfassungsgerichtshof als erstes deutsches Verfassungsgericht die sofortige Aufhebung des Verbots des Verlassens der Wohnung verfügt, für die es aktuell keine belastbaren Gründe mehr gäbe. Die Gerichtsbarkeit erweist sich damit in der Krise - nach einer kurzen Phase der Sprachlosigkeit - insgesamt als fähig, übertriebene Einschränkungen der Grundrechte durch die Exekutiven zu beenden. Bislang wurden über 1.000 Urteile in Corona-Angelegenheiten von deutschen Gerichten gefällt (Siehe Papier, 2020).

${ }^{5}$ Shutdown wird in dieser Studie als temporäre staatlich verordnete und durchgesetzte Einschränkung des öffentlichen Lebens, um eine Epidemie oder Pandemie einzudämmen (Massenquarantäne). Sie umfasst die von einer entweder nationalen oder regionalen Regierung oder Behörde erlassenen Anordnungen an die Bevölkerung, „zu Hause zu bleiben“, um durch zeitweilige Begrenzung oder vollständige Aufhebung der Bewegungsfreiheit der Bevölkerung räumliche Distanzierung innerhalb und außerhalb eines bestimmten Gebiets durchzusetzen. 
Am 25.3. stellt der Bundestag fest, dass durch das neuartige Coronavirus SarsCoV-2 in Deutschland eine epidemische Lage von nationaler Tragweite eingetreten ist. Mit dem am 27.3. in Kraft getretenen „Gesetz zum Schutz der Bevölkerung bei einer epidemischen Lage von nationaler Tragweite" stattete er die Bundesregierung mit weitreichenden zeitlich begrenzten Kompetenzen zur Bewältigung der Krise aus. Bund und Länder haben seit Januar 2020 zur Eindämmung der COVID-19-Pandemie mehr als 350 Gesetze, Verordnungen, Verfügungen und Bekanntmachungen erlassen, darunter Bußgeldkataloge wegen Verstößen gegen die CORONA-Bestimmungen.

\section{Phase III - Auf dem Weg in eine „neue Normalität“}

Bis Mitte April verbessert sich die Lage der Pandemie in Deutschland langsam. Die Zahl der Neuinfektionen ging zurück, was als positiver Effekt der Eindämmungsmaßnahmen seit Mitte März gewertet wurde. Am 15.4. beschließen Bundeskanzlerin und die Regierungschefs der Länder gemeinsam erste Lockerungen des Shutdowns in Deutschland. Schulen sollten z. B. ab 4. Mai schrittweise öffnen, Geschäfte unter 800 qm können ab 20.4. öffnen. Großveranstaltungen bleiben bis Ende August untersagt. Restaurants und Hotels bleiben weiter geschlossen. Das Tragen von Alltagsmasken im öffentlichen Personennahverkehr und beim Einkauf wird ab 27.4. zur Pflicht erklärt. Am 30. April 2020 vereinbarten Bund und Länder weitere Lockerungen. Versammlungen zur Religionsausübung (Gottesdienste und Gebetsveranstaltungen) sollen fortan wieder stattfinden können. Unter Auflagen zur Hygiene, zur Steuerung des Zutritts und zur Vermeidung von Warteschlangen können Museen, Ausstellungen und Galerien, Gedenkstätten sowie zoologische und botanische Gärten wieder geöffnet werden. Weitere Maßnahmen wurden am 6. Mai 2020 beschlossen. Alle Geschäfte dürfen unter Auflagen wieder öffnen. Breiten- und Freizeitsport unter freiem Himmel wird unter Hygiene- und Desinfektionsmaßnahmen und Beibehaltung des Mindestabstands gestattet. Gegen den Widerstand einiger Bundesländer wird ein bundesweiter „Notfallmechanismus" beschlossen, der bis heute in Kraft ist. Die Länder erhalten nunmehr weitgehend die Verantwortung für weitere Lockerungen. Sie verpflichten sich im Gegenzug, sicherzustellen, dass in Landkreisen oder kreisfreien Städten mit kumulativ mehr als 50 Neuinfektionen pro 100.000 Einwohnern innerhalb der letzten 7 Tage ,sofort ein konsequentes Beschränkungskonzept unter Einbeziehung der zuständigen Landesbehörden umgesetzt wird“" (Telefonschaltkonferenz der Bundeskanzlerin mit den Regierungschefinnen und Regierungschefs der Länder am 6. Mai 2020). Der bis zum Redaktionsschluss dieses Beitrages bislang letzte gemeinsame Bund-Länder-Beschluss datiert vom 17. Juni.

Zum Redaktionsschluss dieses Beitrages (Mitte August) ist unklar, ob sich Deutschland bereits in einer weiteren Phase der Pandemiebekämpfung befindet, in der wieder striktere Regelungen erforderlich werden könnten. Die Infektionszahlen in Deutschland und weiten Teilen Europas steigen seit Mitte Juli wieder deutlich an. Nach dem Ende der Sommerferien und dem Schulstart befindet sich das Land aus infektiologischer Sicht in einer schwierigen Phase. Diese Entwicklung wird vom Robert Koch-Institut als sehr beunruhigend angesehen. Es kommt zunehmend zu lokalen Ausbrüchen, 
die erhebliche Ausmaße erreichen können. Sie stehen oft im Zusammenhang mit größeren Feiern oder Freizeitaktivitäten, an Arbeitsplätzen und in Gemeinschaftseinrichtungen. Bundeskanzlerin Merkel bezeichnete die ansteigenden Zahlen von CoronaInfektionen in Deutschland am 15.8. als ,,besorgniserregend, aber noch beherrschbar“ (Deutschlandfunk, 17.8.2020). Sie hat das Vorgehen der Bundesregierung in dieser Phase folgendermaßen beschrieben: „Unser Grundprinzip ist: Wenn Infektionszahlen sinken, dann müssen Grundrechtseingriffe zurückgenommen werden, und wenn Infektionszahlen wie in diesen Tagen wieder steigen, dann müssen Schutzvorkehrungen verstärkt werden“ (Pressekonferenz von Bundeskanzlerin Merkel und NRW-Ministerpräsident Laschet, 18.8.2020). Weitere Lockerungen der Corona-Beschränkungen sind daher augenblicklich nicht zu erwarten, die bestehenden Regeln sollen strikter angewandt werden. Unter allen Umständen wollen Bund und Länder einen erneuten bundesweiten Shutdown mit den wirtschaftlichen und gesellschaftlichen dramatischen Folgen wie im März und April zu vermeiden. Zugleich wächst der Zeithorizont für die Notwendigkeit einer spezifischen Pandemiebekämpfung, der sich immer mehr in das Jahr 2021 hinein verschiebt.

Die wirtschaftlichen Folgen der Pandemie und des Shutdowns in Deutschland geraten nunmehr stärker in den Blick. Bis Mitte März hatte die Bundesregierung Rettungsmaßnahmen für die Abfederung der wirtschaftlichen Folgen der Corona-Krise in Höhe von mehr als 1,2 Billiarden Euro bewilligt. Diese gigantische Summe setzt sich aus dem Nachtragshaushalt des Bundes für 2020 in Höhe von 156 Milliarden Euro, dem neu geschaffenen Wirtschaftsstabilisierungsfonds und dem erweiterten Garantierahmen für die Kreditanstalt für Wiederaufbau (KfW) zusammen (Stand 23.3.). Allein in diesem Jahr plant der Bund mit zusätzlichen Schulden in Höhe von 156 Milliarden Euro. Nimmt man alle Rettungspakete zusammen, beläuft sich das Volumen aus Garantien und Krediten auf über 1,8 Billionen Euro (Schätzung 18.4.2020).

Zusätzlich haben die Bundesländer solche Rettungsprogramme aufgelegt. Zugleich beginnt die politische Auseinandersetzung über die politischen Konzepte zur Überwindung der Corona-Krise wieder, die Opposition beginnt die Regierung zu kritisieren, erste Demonstrationen finden wieder statt. Deutschland ist auf dem Weg in eine ,neue“" Normalität, (noch) nicht zurück in jene vor dem März 2020. Neben dem Hochfahren der Wirtschaft und dem Öffnen der Gesellschaft bleibt die Pandemie real, solange kein wirksamer Impfstoff gefunden worden ist. Die politischen Unwägbarkeiten bleiben daher sehr hoch.

Als wichtiger Faktor in der Krise erwies sich die Akzeptanz der Bevölkerung für die drastischen Maßnahmen von Bund und Ländern zur Eindämmung der Pandemie. Gut einschätzen lässt sich deren Verlauf anhand eines speziellen „CORONA-Monitors", den das Bundesinstitut für Risikobewertung seit dem 24. März herausgibt, die letzte erfasste Ausgabe ist die vom 18./19.8. Danach bewertete auf dem bisherigen Höhepunkt der COVID-19-Pandemie im März zwischen 97\% (Schließung von Kultureinrichtungen und Absage von Veranstaltungen) und 74\% (Ausgangssperre) der Befragten konkrete Maßnahmen von Bund und Länder als angemessen (Nach Bundesinstitut für Risikobewertung 2020, S. 16) Bis Mitte Juni sanken diese Akzentranzwerte jeweils um bis zu 20\% ab, danach siegen diese wieder an befinden sich aber weiter auf hohem Niveau. 
Das Krisenmanagement der Regierungen in Bund und Ländern, einschließlich des Shutdowns, von einer klaren Mehrheit der Deutschen positiv bewertet. Der Shutdown findet eine klare Zustimmung. In der Krise sind positiven Bewertung des Regierungshandeln angesichts des Coronavirus angestiegen. Nach den wöchentlichen YouGovUmfragen stieg der Anteil der Befragten, der der Meinung war, die Regierung gehe sehr oder eher gut mit dem Coronavirus um von 52\% (16. März) auf 62\% (23. März) und dann auf 70\% (30. März). Dieser Wert wird auch am 27. April noch gemessen (Daten nach YouGov 2020). Die Umfragen der Meinungsforschungsinstitute zeigen gegen Ende April eine sinkende Akzeptanz der Bevölkerung und mehr Druck zur Beendigung des Shutdown.

Obwohl die Befragten den Föderalismus laut einer Umfrage der Universität Konstanz vom März/April 2020 eher als ungeeignet für ein wirksames Krisenmanagement ansehen, unterstützen sie dennoch die Möglichkeit regionaler Maßnahmen. „Gerade die Regierungsebenen, die in der Krise besonders aktiv sein können, profitieren davon. Dies sind insbesondere die Kommunen und die Landesbehörden " (Eckhard, Lenz 2020, S. 2). Dies gilt jedoch offenbar eher für die Lockerung in weniger betroffenen Landkreisen oder Städten als für schwierige Situationen, in denen die Anzahl der Infektionen wieder zunehmen kann.

\section{Fazit}

Deutschland gehört zweifellos zu den am stärksten von der CORONA-Pandemie betroffenen Länder weltweit. Angesichts einiger positiver Indikatoren (Bislang vergleichsweise relativ wenigen Corona-Tote, einer sehr hohe Zahl von Genesenden und einen funktionsfähigen stabilen Gesundheitssystem), kann man dennoch im internationalen Vergleich von einem gelungenen Krisenmanagement sprechen. Deutschlands Regierungen in Bund und Ländern erwiesen sich insbesondere nach dem Shutdown als handlungsfähig. Den politischen Entscheidern in Bund und Ländern gelang es nach anfänglichem Zögern, die Balance zwischen gesundheitlichen sowie wirtschaftlichen Erwägungen zu finden. Dieser Kurs wurde bislang von der überwiegenden Mehrheit der Bevölkerung mitgetragen, die durch eine hohe Einsicht in die Notwendigkeit des Shutdowns und große Disziplin bei der Befolgung der neuen Regeln einen wesentlichen Beitrag zur Eindämmung der CORONA-Krise geleistet hat. Diese Unterstützung beginnt allerdings nunmehr zu bröckeln. Neben den fortgesetzten Bemühungen, das Virus einzudämmen, gewinnen daher die Überwindung der wirtschaftlichen und gesellschaftlichen Folgen des Shutdown an Bedeutung.

\section{CORONA-Krisenmanagement der deutschen Kommunen}

Die deutschen Kommunen waren durch die erfolgreiche Bewältigung verschiedener Gesundheitsgefährdungen wie SARS im Jahr 2003, Ebola, EHEC, der Vogelgrippe 2006 oder der Schweinegrippe von 2009 im Umgang mit Katastrophen durchaus erprobt. Wichtige Hilfestellung brachte auch der Nationale Pandemieplan von 2017 
sowie Politprojekte für den Pandemiefall in einzelnen Kommunen, so z. B. in der Stadt Dortmund. Trotz dieser Erfahrungen war der öffentliche Gesundheitsdienst in Deutschland nicht gut auf die Pandemie vorbereitet. Die Zahl der Ärzte in den 375 deutschen Gesundheitsämtern war seit dem Jahr 2000 wegen Personalabbaus, Fachkräftemangel und nicht besetzter Stellen um ca. 1/3 zurückgegangen. Ende 2018 waren noch rund 2.500 Ärzte sowie 17.000 Mitarbeiter in kommunalen Gesundheitsämtern tätig. Damit waren diese nicht ausreichend in der Lage, ihren gesetzlichen Aufgaben nachzukommen. Eine pandemische Infektionswelle wie bei COVID-19 war allerdings auch für den kommunalen Katastrophenschutz völlig neu.

Alle Kommunen in Deutschland sind von der Corona-Krise direkt und indirekt betroffen, die gilt sowohl für die Landkreise und kreisfreien Städte als auch für die Gemeinden. Allerdings sind regionale Unterschiede zu beobachten. Neben Großstädten finden sich die etwa zwei Dutzend Pandemie-Hotspots in Deutschland fast ausschließlich in Süd- und Westdeutschland, während deren Ausbreitung im Nord- und Ostdeutschland (bislang) deutlich geringer ist. Die Fallzahl betrug nach RKI-Angaben am 15.8. 268 Fälle pro 100.000 Einwohner, wobei das Bundesland Bayern mit 404 Fällen am stärksten betroffen war und das dünnbesiedelte Bundesland Mecklenburg-Vorpommern mit 60 Fällen am wenigsten. Auch auf Kreisebene findet sich diese Differenzierung, die Pandemie-Hotspots fanden sich Mitte August in Bayern, Thüringen, Niedersachsen und Hessen. Damit ist der Handlungsdruck bei der Pandemiebekämpfung in den Kommunen durchaus unterschiedlich (Daten nach Robert-Koch-Institut, 2020).

Ähnlich wie bei Bund und Ländern sahen sich auch die Kommunen gezwungen, im Februar/März 2020 innerhalb kürzester Zeit in einen spezifischen Corona-Modus zu wechseln. In der Covid-19 Pandemie können die deutschen Kommunen vor allem zwei Kompetenzen zurückgreifen: (1) Sie sind laut Infektionsschutzgesetz meist für die Implementation der von Bund und Ländern beschlossenen Gesetze, Verordnungen usw. vor Ort verantwortlich. (2) Sie können als Gebietskörperschaften aber auch eigene Maßnahmen zur Bekämpfung der Pandemie vor Ort treffen (z. B. als befristete Allgemeinverfügung).

Die Kommunen verfolgten bei der COVID-19-Pandemie-Bekämpfung drei Strategien: Die Strukturanpassungsstrategie war vom ersten Tag der Pandemie an notwendig und wird während des gesamten Verlaufs der Krise notwendig bleiben. Die Netzwerkstrategie ist auch eine dauerhafte Notwendigkeit für die Kommunen, durch eine verbesserte Interaktion und Kommunikation mit relevanten organisationsinternen Akteuren sowie mit den Akteuren im Verwaltungsumfeld einen besseren und vor allem schnelleren Zugang zu Informationen, Wissen und Ressourcen zu ermöglichen. Die Reflexionsstrategie wird im Verlauf der Pandemie immer wichtiger, vor allem als permanenter Lern- und Bewertungsprozess zur Anpassung des lokalen Pandemiemanagements an die veränderte Infektionssituation.

Im Folgenden sollen die wichtigsten kommunalen Anpassungsleistungen während der CORONA-Krise in fünf Bereichen herausgearbeitet werden: bezüglich der Verwaltungsführungen, der Kernverwaltungen, der kommunalen Unternehmen, der Kommunalpolitik und der lokalen Bürgerbeteiligung (Siehe auch Pöhler et al., 2020).

- Verwaltungsführung: Die CORONA-Krise ist auch auf lokaler Ebene die „Stunde der Exekutive“, d. h. Landräte, Oberbürgermeister und Bürgermeister als Haupt- 
verwaltungsbeamte und Leiter kommunaler Verwaltungen sind gefordert. Sie tragen die Gesamtverantwortung für das lokale Krisenmanagement und wirken an der Kooperation im jeweiligen Bundesland entscheidend mit. Die in der Krise nötige „Führung auf Distanz“ erforderte rasche Lernprozesse der Hauptverwaltungsbeamten. Seit Mitte Februar wurden in allen Landkreisen und kreisfreien Städten spezielle Corona-Krisenstäbe gebildet (siehe oben). Seit Ende April werden diese wieder aufgelöst, eine Reaktivierung bleibt möglich.

- Kernverwaltungen: Auch diese mussten sich rasch auf die Herausforderungen der Pandemie einstellen und durch rasche betrieblich-organisatorische Veränderungen die Handlungsfähigkeit der Kommunalverwaltungen in der Krise sicherzustellen. Komamunale Ressourcen mussten umverteilt werden, vor allem auf das Gesundheitsamt. Hinzu kamen baulich-funktionelle Anpassungen in kommunalen Gebäuden, um das Infektionsrisiko sowohl für Mitarbeiter als auch Bürger bzw. Kunden zu minimieren. Mitte März mussten alle Ämter für den Publikumsverkehrs geschlossen werden, darunter Bürgerämter, kommunaler Bibliotheken, Volkshochschulen, Archive. Sprechstunden wurden abgesagt, Rathäuser für den Publikumsverkehr geschlossen. Anfang Mai wurden die Rathäuser wieder zu den regulären Öffnungszeiten für den allgemeinen Publikumsverkehr geöffnet, nunmehr aber mit strengen Abstands- und Hygieneregeln. Die Bürger werden gebeten, ihr Anliegen weiterhin möglichst per E-Mail oder telefonisch zu erledigen. Viele Kommunen weiteten kurzfristig ihr digitale Serviceangebot für Bürger und Unternehmen aus.

- Gesundheitsämter: Vor allem die von einem Amtsarzt geleiteten kommunalen Gesundheitsämter sind in der Krise stark gefordert. Diese sind verantwortlich, örtliche Infektionsfälle zu registrieren, deren Infektionsketten nachzuvollziehen sowie die häusliche Quarantäne von Patienten mit milden Verlaufsformen von Covid-19 zu kontrollieren. Schließlich müssen sie die Verwaltungsführung und die Kommunalpolitiker in diesem komplexen Politikfeld fachgerecht beraten. Deren Arbeitsaufwand war vor allem im März bis Mitte April außerordentlich hoch, viele arbeiteten am Kapazitätslimit. Dies zeigt sich auch noch heute (Mitte August), wenn ein Landkreis über Nacht zum COVID-19 Hotspot wird. Seit Mitte April entspannt sich die Lage in den meisten Gesundheitsämtern, insgesamt haben sich diese nach Einschätzung von Helmut Dedy, Hauptgeschäftsführer des Deutschen Städtetags „organisatorisch sehr gut auf die enormen Herausforderungen eingestellt“ (Frankfurter Allgemeine Zeitung, 17.4.2020). Seit Ende Juli 2020 nehmen die Belastungen für die Gesundheitsämter wieder zu.

- Andere Ämter: Auch bei anderen kommunalen Ämtern nahm die Arbeitsbelastung während der Krise stark zu. Gemeinsam mit der Polizei, die in Deutschland Teil der Landesverwaltung ist, mussten die kommunalen Ordnungsämter kurzfristig neue Aufgaben übernehmen, z. B. die Kontrolle der Einhaltung der Quarantänebestimmungen, Ausgangsbeschränkungen sowie entsprechender CORONA-Bußgeldkataloge der Länder. Während des Shutdowns mussten die Jugendämter unter schwierigen Umständen dafür Sorge tragen, dass die familiäre Gewalt nicht zunimmt. Der Dienst kommunaler Bauaufsichtsbehörden und anderer Genehmigungsbehörden war zwischen Mitte März und Ende April eingeschränkt. Dadurch können Beteiligungs- und Bearbeitungsfristen der Genehmigungsverfahren teilweise nicht einge- 
halten werden, was zu zeitlichen Verzögerungen und rechtlichen Problemen führte, z. B. bei Kfz-Zulassungen und Baugenehmigungen.

- Kommunalfinanzen: Rasch war klar, dass die Pandemie in allen deutschen Kommunen unmittelbar zu gravierenden finanziellen Belastungen führen wird. Erstens brechen viele geplante kommunale Einnahmen weg. Erhebliche Einbrüche werden vor allem bei der Gewerbesteuer als wichtigste kommunale Einnahmequelle erwartet. Weitere kommunale Einnahmenverluste werden bei der Einkommensteuer, den Kitabeiträgen sowie den Einnahmen bei Bibliotheken, Schwimmbädern und Theatern erwartet. Neben den Kommunen selbst gerieten auch deren Unternehmen wie z. B. Verkehrsbetriebe durch die Pandemie in finanzielle Schwierigkeiten. Kommunen rechnen daher für das Jahr 2020 mit geringeren Ausschüttungen ihrer Beteiligungen oder müssen sogar deren Verluste ausgleichen. Andererseits entstehen den Städten und Gemeinden durch die Pandemie viele direkte und indirekte Zusatzkosten, deren Gesamthöhe noch nicht abzuschätzen ist. Dies betrifft z. B. die Ressourcen für die kreislichen CORONA-Krisenstäbe, die neuen Aufgaben der kommunalen Gesundheitsämter, die Vorleistungen für mobile Corona-Teststationen, die Anschaffung von Schutzkleidung und Gesichtsmasken für kommunales Personal sowie den IT-Bereich, der schneller als geplant ausgebaut werden muss. Es zeichnet sich auch höhere kommunalen Sozialausgaben ab.

Mittlerweile haben Bund und Länder finanzielle Unterstützung für die Kommunen zugesagt. Im Rahmen des im Juni 2020 vom Bundestag verabschiedeten „Konjunkturpakets“" zur Ankurblung der Wirtschaft in Höhe von 130 Mrd. Euro ist auch ein „Kommunaler Rettungsschirm“ beschlossen worden. Dieser sieht finanzielle Unterstützung des Bundes für bereits zur Umsetzung geplante kommunale Investitionen vor, die wegen der Corona-Krise kurzfristig vor Ort nicht realisiert werden konnten. Dies gilt auch für kommunale Zukunftsinvestitionen in die Digitalisierung der Schulen, die Verkehrswende und in klimafreundliche Gebäude und Versorgung. Der Bund erhöht seinen Anteil für die Kosten der Unterkunft für Empfänger von Sozialleistungen von 50\% auf 75\%. Der Bund wird die Hälfte der Ausfälle der kommunalen Gewerbesteuer übernehmen sowie die Verluste der kommunalen Verkehrsbetriebe in der Corona-Krise ausgleichen.

- Kommunale Unternehmen: Auch kommunale Unternehmen und Betriebe sind von der Krise stark getroffen (Siehe EY Public Services Sonder-Newsletter, April 2020). Verkehrsbetriebe, Theater, Museen, Bäder, Kultur- und Sporteinrichtungen und andere Einrichtungen wurden während des Shutdowns geschlossen und hatte keine Einnahmen mehr. Seit Ende April werden diese schrittweise wieder geöffnet, aktuell sind nur noch wenige geschlossen. Durch die neuen CORONAAbstands- und Hygieneregeln ist deren Nutzerzahl aber deutlich geringer. Kommunalen Versorgungsunternehmen hingegen waren nur wenig von der Krise betroffen. Zahlreiche kommunale Netzbetriebe mussten Investitionen aussetzen. Ein bis Ende 2020 befristeter Tarifvertrag zur Regelung der Kurzarbeit (TV COVID) soll negative Folgen der CORONA-Krise für kommunale Unternehmen mildern. Betriebsbedingte Kündigungen sollen damit verhindert, Urlaubsansprüche erhalten sowie Einkommen gesichert werden. Dies soll auch dem Fortbestand kommunaler Einrichtungen dienen. Für die weitaus meisten Bereiche des öffentlichen Dienstes 
in den Kommunen spielt Kurzarbeit allerdings kaum eine Rolle, z. B. Krankenhäuser, Pflegeeinrichtungen, Kinderbetreuung und den Sozial- und Erziehungsdienst. Es gelang den kommunalen Unternehmen in der Corona-Krise bislang, eine stabile Versorgung der Bevölkerung mit den Leistungen der Daseinsvorsorge (Wasser, Abwasser, ÖPNV usw.) zu sichern.

- Kommunalpolitik: Mit dem Shutdown des öffentlichen Lebens im Zuge der CORONA-Pandemie Mitte März 2020 wurde die Kommunalpolitik in Deutschland faktisch lahmgelegt. Um dennoch die demokratische Mitsprache der Bürger und die Kontrolle der Verwaltung durch die kommunalen Parlamente sicherzustellen, waren auch hier rasche Anpassungen erforderlich. Zwischen Anfang März und Mitte Mai fanden nur wenige Sitzungen der ehrenamtlichen Kommunalparlamente oder ihrer Ausschüsse statt. Wegen der gesetzlich vorgeschriebenen Präsenzpflicht wurden diese wegen des CORONA-Infektions-risikos und der Ausgangsbeschränkungen abgesagt oder waren beschlussunfähig. Wichtige Beschlüsse wurden daher von Verwaltungsbeamten per Eilentscheidungen getroffen.

Um diesen Zustand schnell zu beenden beschlossen viele Bundesländer „Kommunale Notlagegesetze“, um die Kommunalparlamente auch in der Corona-Pandemie handlungsfähig zu halten. Der Brandenburger Landtag beschloss am 15.4. ein bis Ende September 2020 befristetes Gesetz (GVB1. Bbg. I/20, [Nr. 14]), nachdem kommunale Volksvertretungen befristet auch per Video- und Telefonkonferenz tagen können. In Ausnahmefällen können diese Entscheidungen auf die kleineren Hauptausschüsse übertragen oder im schriftlichen Umlaufverfahren fassen. Um die Öffentlichkeit zu gewährleisten, sollen deren Beratungen im Livestream auf der Homepage der Gemeinde oder in einem gesonderten Raum der Verwaltung verfolgt werden können. Seit Ende April begannen die Wiederaufnahme des kommunalpolitischen Lebens. Zumeist tagen zuerst die kleineren Hauptausschüsse, später die größeren Kommunalparlamente tagen können, allerdings wegen des notwendigen Sicherheitsabstandes in größeren Räumlichkeiten. Auch deren Geschäftsordnungen müssen angepasst werden.

Betroffen von der Corona-Pandemie waren auch die anstehenden Kommunalwahlen im Bundesland Bayern. Der erste Wahlgang fand am 15. März ohne besondere Berücksichtigung der Pandemie-Lage statt, was vielfach kritisiert wurde. Die erforderlichen Stichwahlen am 29. März wurden nach einer Gesetzesänderung als reine Briefwahl durchgeführt. Besonders betroffen von der CORONA-Krise sind auch die Ortsvereine politischer Parteien und die Organisationen von Bürgerinitiativen, die für die lokale Demokratie besonders wichtig sind. Angesichts der zeitweiligen Versammlungsverbote und Kontaktsperre während des Shutdowns mussten diese mit ihren Mitgliedern online kommunizieren.

- Lokale Bürgerpartizipation: Auch die Bürgerbeteiligungsverfahren z. B. an der gemeindlichen Bauleitplanung waren von der Pandemie betroffen. Entwürfe von Bauleitplänen müssen gesetzlich durch die Kommunen für mindestens 30 Tage in Papierform öffentlich ausgelegt und im Internet bereitgestellt werden. Bürger müssen auch in Zeiten der CORONA-Krise die Möglichkeit haben, sich über Planungen zu informieren und zu diesen zu äußern. Unproblematisch ist dabei die digitale Information im Internet über die Homepage der Gemeinden. Schwieriger ist es, 
den analogen Zugang zu diesen Planungsunterlagen zu sichern, der jedoch weiter nötig ist, da nicht alle Bürger Internet-Zugang besitzen. Nach vorheriger telefonischer Meldung stellen Kommunen den Bürgern im Rathaus einen separater Raum zur Verfügung, um die Unterlagen einsehen zu können. Fragen zu kommunalen Planungen können weiterhin auch telefonisch gestellt werden. Die Auslegungsfristen können verlängert werden. Die öffentliche Auslegung noch nicht begonnener Verfahren wurde verschoben, seit Ende Mai findet diese wieder statt.

In einigen deutschen Städten wird im Zuge der Krise mit online-Bürgerversammlungen experimentiert, da traditionelle Präsenzveranstaltungen im März/April nicht möglich waren. Da der Informations- und Kommunikationsbedarf der Bürger weiter sehr hoch ist, müssen barrierefreie, für den Bürger kostenfreie und datensichere technische Lösungen gefunden werden. So wurde in der Stadt Tengen (Land Baden-Württemberg) mit 4.670 Einwohnern eine für März geplante Präsenzbürgerversammlung online veranstaltet. Auch die virtuelle Organisation der lokalen Bürgergesellschaft erlebt in der CORONA-Krise einen Schub. Entweder die lokale Zivilgesellschaft organisiert sich auf diese Weise selbst, oder Kommunen stellen ein solches Angebot zur Verfügung, wie z. B. die Stadt Bühl in Baden-Württemberg mit 29.000 Einwohnern mit dem Projekt "Palim Palim". Viele solcher OnlineProjekte sind seit Ende Februar entstanden, um lokale Hilfs- und Unterstützungsangebote für spezielle Bevölkerungsgruppen, die von der CORONA-Pandemie besonders betroffen sind, zu bündeln. Allerdings bestehen in 75\% der Kommunen solche digitalen Beteiligungsangebote bisher nicht (Berliner Institut für Partizipation 2020).

\section{Fazit und Ausblick}

Zusammenfassend ist mehr als ein halbes Jahr, nachdem COVID-19 Deutschland erreicht hat, folgendes Zwischenfazit zu ziehen. Ein Ende der Pandemie nicht abzusehen. Einerseits gehört Deutschland zu den weltweit an den stärksten betroffenen Ländern, andererseits kann sein Krisenmanagement zur Eindämmung der Pandemie als bislang gelungen bezeichnet werden. Deutschlands Regierungen in Bund und Ländern erwiesen sich als handlungsfähig, die überwiegende Mehrheit der Bevölkerung trug diesen Kurs bislang mit. Dennoch bleibt auch die weitere politisch-administrative Steuerung in der Krise mit immer noch unvollständigem Wissen über das Virus und mit vielfältigen gesundheitlichen, sozialen und wirtschaftlichen Zielkonflikten sehr schwierig. Ein Ende der Krise ist wohl erst erreicht, wenn ein Impfstoff oder ein vergleichbarer Schutz der Bevölkerung vorliegt.

Die eingangs genannter, theoretischer politikwissenschaftlicher Fragestellung dieses Beitrages lässt sich klar beantworten. Die dezentrale, föderale Struktur hat sich überwiegend positiv auf die Pandemiebekämpfung und das Krisenmanagement ausgewirkt. Dazu hat auch beigetragen, dass in den verschiedenen Phasen der Pandemiebekämpfung das Verhältnis zwischen zentralem und dezentralem Krisenmanagement angepasst wurde. Anfangs dominierte eher ein dezentrales, auf Hotspots fokussiertes Krisenmanagement. Während des Lockdowns im März/April bekam die zentrale Ko- 
ordinierung und Abstimmung zwischen Bund und Ländern eine höhere Bedeutung. Seither liegt der Schwerpunkt wieder auf einer dezentralen Eindämmungsstrategie auf der Grundlage eines gemeinsamen Entscheidungsrahmens. Seit Anfang August 2020 mehren sich wegen der steigenden Infektionszahlen wieder die Stimmen für ein stärker zentralistisches Vorgehen.

Die in der Krise ist in Deutschland die Akzeptanz für die Rolle des Staates als aktiver, regulierender und fürsorglicher Akteur deutlich gewachsen. Ob dies mittel- oder langfristig zu einer sektoralen Zentralisierung in Deutschland z. B. im Gesundheitsoder Bildungssystem führen wird, ist offen.

Die Kommunen in Deutschland haben sich als Rückgrat der Bewältigung in der CORONA-Krise bewährt. Den Landkreisen, kreisfreien Städte und alle anderen Gemeinden gelang es, die Entscheidungen des Bundes und der Länder administrativ rasch und effektiv umzusetzen. Zugleich nutzten viele von ihnen ihre Autonomie, um eigene, den örtlichen Umständen angepasste Beiträge zur Eindämmung der Ausbreitung von COVID-19 zu leisten. Die föderale, dezentrale Struktur der Bundesrepublik hat eine weitere Bewährungsprobe bestanden. Insgesamt haben die deutschen Kommunalverwaltungen ihre institutionellen Kapazitäten bisher recht gut an die Herausforderungen im Zusammenhang mit COVID-19 angepasst. Der Anpassungsprozess wurde durch ihre territorial ausgerichtete institutionell Mehrzweckorganisation unter einheitlicher Leitung erleichtert. Diese ermöglichte es, schnell Anpassungen vorzunehmen, Ressourcen über Branchengrenzen hinweg zu verlagern und die Corona-bedingten Gesundheitsstandards in allen lokalen Dienstleistungen umzusetzen.

Einige Schwächen der lokalen Gebietskörperschaften konnten ebenfalls festgestellt werden: Am Anfang betrifft dies hauptsächlich die unzureichende personelle und technische Ausstattung der örtlichen Gesundheitsbehörden. Viele bestehende kommunale Rechtsvorschriften waren nicht auf die Herausforderungen einer Pandemie vorbereitet, z. die Verpflichtung, physisch an den Sitzungen des Gemeinderats teilzunehmen. Die Kommunen wurden zu oft durch Entscheidungen der Länder-Krisenteams mit vollendeten Tatsachen konfrontiert.

Die COVID-19 Pandemie und deren Folgen für die Gesundheit der Bevölkerung, deren gesellschaftlichen Zusammenhalt und die Volkswirtschaften werden viele neue Forschungsfragen auf. Die Politikwissenschaft kann einen Beitrag dazu leisten, die dabei auftretenden Zielkonflikte und Anpassungsprobleme genauer zu analysieren, damit Staat und Gesellschaft für künftige Krisen, vor allem die Klimakrise, besser gerüstet sind.

\section{Literatur}

Behnke, N. (2020), Föderalismus in der (CORONA-)Krise?, in: Aus Politik und Zeitgeschichte, 70. Jahrgang, 35-37/2020, 24. August 2020, S. 9-15.

Berliner Institut für Partizipation (2020), Das verlorene Jahr? Auswirkungen der Corona-Pandemie auf die Bürgerbeteiligung in Deutschland, Berlin.

Brandenburgisches Ministerium für Soziales, Gesundheit, Integration und Verbraucherschutz (2020), Pressemitteilung vom 17.3.2020.

Bundesinstitut für Risikobewertung (2020), Corona-Monitor, 18./19.8., S. 16. 
Bundesregierung (2020a), Regierungshandeln in Zeiten von Covid-19, 9. April 2020.

Bundesregierung (2020b), Besprechung der Bundeskanzlerin mit den Regierungschefs der Länder, 22.3.2020.

Eckhard St., Lenz A. (2020), Die öffentliche Wahrnehmung des Krisenmanagements in der Covid-19 Pandemie, S. 2.

Franzke J. (2020), German Municipalities in the COVID-19 Pandemic Crisis. Challenges and Adjustments. A preliminary analysis. In IGU Commission Geography of Governance (2020). Local Government Response Towards Covid-19 Pandemic: a Worldwide Survey and Comparison, Working paper 1, https://sites.google.com/view/igucgog-covid19/working-papers.

Fuhr H., Fleischer J., Kuhlmann S. (2018), Federalism and decentralization in Germany. Basic features and principles for German development cooperation, GIZ.

Gesetz zur Sicherstellung der Handlungsfähigkeit der brandenburgischen Kommunen in außergewöhnlicher Notlage vom 15.4.2020 (GVBl. Bbg. I/20, [Nr. 14]).

Gesetz zur Verhütung und Bekämpfung von Infektionskrankheiten beim Menschen (Infektionsschutzgesetz - IfSG) vom 20.07.2000 (BGBl. I S. 1045), zuletzt geändert am 27. März 2020 (BGBl. I S. 587).

Gesetz zum Schutz der Bevölkerung bei einer epidemischen Lage von nationaler Tragweite (COVIfSGAnpG) vom 27.03.2020, BGB1. I S. 587 (Nr. 14).

Hesse K. (1962), Der unitarische Bundesstaat, Müller, Karlsruhe.

Kuhlmann S. (2020), Between Unity and Variety: Germany's Responses to the COVID-19 Pandemic, in: The COVID-19 Pandemic: Early Lessons for Public Governance, Eds. P. Joyce, F. Maron, S. R. Purshottama, IIAS Special Report. Brussels.

Kuhlmann S., Wollmann H. (2019), Introduction to Comparative Public Administration: Administrative Systems and Reforms in Europe, Second edition, Edward Elgar, Cheltenham-Northampton.

Papier H.-J. (2020), Umgang mit der Corona-Pandemie: Verfassungsrechtliche Perspektiven, in: Aus Politik und Zeitgeschichte, 70. Jahrgang, 35-37/2020, 24. August 2020, S. 4-8.

Pöhler J., Bauer M. W., Schomaker R. M., Ruf V. (2020), Kommunen und COVID-19: Ergebnisse einer Befragung von Mitarbeiter*innen deutscher Kommunalverwaltungen im April 2020, Speyer (Speyer Arbeitshefte, Nr. 239).

Pressekonferenz von Bundeskanzlerin Merkel, Bundesgesundheitsminister Spahn und RKI-Chef Wieler (2020), 11.3.2020.

Presse- und Informationsamt der Bundesregierung (2020a), Maßnahmen der Bundesregierung zur Eindämmung der COVID-19-Pandemie und zur Bewältigung ihrer Folgen, 30. April 2020.

Presse- und Informationsamt der Bundesregierung (2020b), Vereinbarung zwischen der Bundesregierung und den Regierungschefinnen und Regierungschefs der Bundesländer angesichts der Corona-Epidemie in Deutschland, 16. März 2020.

Robert-Koch-Institut (2020), Täglicher Lagebericht zur Coronavirus-Krankheit-2019 (COVID-19), 15.8.2020.

Runderlass des NRW-Ministeriums für Inneres und Kommunales (2016), Krisenmanagement durch Krisenstäbe im Lande Nordrhein-Westfalen bei Großeinsatzlagen, Krisen und Katastrophen, Düsseldorf.

Schmidt M. G. (2015), Policy-Diversität im deutschen Föderalismus, https://www.uni-heidelberg. $\mathrm{de} / \mathrm{md} /$ politik/personal/schmidt/conclusion___policy_diversity_in_german_federalism_-_ main_document.pdf.

YouGov (2020), COVID-19-Trackerergebnisse, Ende April, https://yougov.de/news/2020/04/29/ covid-19-trackerergebnisse-ende-april-leicht-sinke/. 


\title{
Zusammenfassung
}

Dieser Beitrag befasst sich mit der Analyse und der Bewertung des Krisenmanagements in Deutschland im Zuge der COVID-19 Pandemie (Redaktionsschluss Mitte August 2020). Schwerpunkt bilden die Analyse und kritische Bewertung der Kompetenzverteilung zwischen Bund, Ländern und Kommunen in Deutschland bei Gesundheitskrisen wie dieser. Rechtliche Basis dafür ist die allgemeine Generalklausel des Nationalen Infektionsschutzgesetzes (IfSG), die weitgehende Eingriffe in das öffentliche Leben erlaubt, ohne den Notstand ausrufen zu müssen. Im nächsten Schritt werden die bisherigen Phasen der Pandemiebekämpfung in Deutschland kurz dargestellt. Als Fallbeispiel werden schließlich die Anpassungsstrategien der Kommunen während der CORONA-Krise bezüglich der Verwaltungsführungen, der Kernverwaltungen, der kommunalen Unternehmen, der Kommunalpolitik und der lokalen Bürgerbeteiligung herausgearbeitet. Der Beitrag endet mit einem Fazit, welches vorläufig bleiben muss, da die Pandemie noch nicht vorüber ist.

Keywords: CORONA-Krise, Deutschland, Föderalismus, Krisenmanagement, Öffentliche Verwaltung

(Redaktionsschluß Mitte August 2020)

\section{Germany,s Crisis Management in the CORONA-Pandemic. Challenges of a Federal Political-Administrative System}

\begin{abstract}
Summary
This article deals with the analysis and evaluation of crisis management in Germany in the course of the COVID-19 pandemic (deadline mid-August 2020). The focus is on the analysis and critical assessment of the distribution of competencies between the federal, federal states and local levels in the German political-administrative system in health crises like this pandemic. The legal basis for the state measures is is the general clause of the National Infection Protection Act (IfSG), which allows extensive interventions in public life without having to declare a state of emergency. In the next step, the previous phases of fighting the pandemic in Germany are briefly presented. Finally, as a case study, the adaptation strategies of the municipalities during the CORONA crisis with regard to administrative management, core administrations, municipal companies, municipal politics and local citizen participation are worked out. The article ends with some conclusions, which must remain for the time being, as the pandemic is not over yet.
\end{abstract}

Key words: CORONA crisis, Germany, federalism, crisis management, public administration

(Deadline mid-August 2020) 